\title{
Terapia Hormonal da Menopausa
}

\section{mini-revisão}

\section{DOLORES PARDINI}

\author{
Disciplina de Endocrinologia, \\ Universidade Federal de São \\ Paulo/ Escola Paulista de \\ Medicina, São Paulo, SP.
}

Embora a reposição estrogênica esteja disponível há mais de 6 décadas, as mulheres e mesmo os profissionais da saúde estão confusos pelas opiniões divergentes em relação aos riscos e benefícios da terapia hormonal na menopausa (THM), estrogênica (TE) ou estro-progestagênica (TEP). A principal indicação para terapêutica hormonal na menopausa é o alívio dos sintomas menopausais, tais como sintomas vasomotores, alterações gênito-urinárias e a prevenção de osteoporose nas pacientes de risco. Em outras áreas de pesquisa, principalmente ao que se refere aos efeitos nos sistemas cardiovasculares e nervoso central, os resultados atuais na literatura são conflitivos. $O$ tratamento por mais de 5 anos não adiciona risco significativo para câncer de mama, mas diminui significativamente o risco de fratura osteoporótica. Algumas mulheres podem ser susceptíveis a risco tromboembólico precoce, mas quando a TH for adequada após avaliação individualizada, os benefícios superam os riscos e o tratamento deve ser recomendado. Estudos futuros são necessários para identificar novas indicações para TH e diminuir ou abolir seus riscos. A pesquisa clínica continua na identificação de fatores genéticos que possam influenciar a resposta individual à $\mathrm{TH}$, diferentes formulações estrogênicas, diferentes vias de administração e liberação, além das opções de dose. Nas mulheres que apresentam os sintomas da síndrome climatérica de forma severa durante a peri e pós-menopausa já existem evidências conclusivas oriundas de vários estudos randomizados controlados de que a TH é a única terapia com resultados satisfatórios. Os médicos devem sempre fazer suas decisões terapêuticas com base nos riscos e benefícios individuais de cada paciente, tendo a responsabilidade e o dever de promover as condições para a mulher atravessar a transição menopáusica com qualidade de vida. (Arq Bras Endocrinol Metab 2007;51/6:938-942)

Descritores: Menopausa; Terapia hormonal; Climatério

\section{ABSTRACT}

Menopausal Hormone Therapy.

Although estrogen has been clinically available for more than 6 decades, women have been confused by different opinions regarding the risks and benefits of menopausal hormone therapy (HT), estrogen therapy (ET), and estrogen-progestin therapy (EPT). The main indication for HT use in postmenopausal women remains the relief of vasomotor symptoms and vulvovaginal atrophy, and is effective in the prevention of osteoporosis. In other areas of research, notably in cardiovascular and central nervous system effects, the recent literature has produced conflicting results. Treatment for up to 5 years does not add significantly to lifetime risk of breast cancer, but significantly decreases bone loss and risk of osteoporotic fractures. Some women may be susceptible to early thrombotic risk, but when appropriate HT is given after individual clinical evaluation, the benefits will far outweigh any potential risks and the treatment should be recommended. Clinical research continues into genetic factors influencing the response to ET/HT, different estrogen formulations, different modes of delivery and lower-dose options. Patients and clinicians should make treatment decisions on the basis of an individual's needs and risks, and should enhance a woman's ability to undergo the menopausal transition with minimal disruption to her quality of life. In women experiencing distressing climacteric symptoms during the peri and postmenopause there is conclusive evidence from abundant randomized controlled trials that systemic hormone therapy (HT) of any type affords symptom relief, with no alternative treatment producing similar effect. Future research is needed to identify new indications for HRT and to diminish or abolish its potential risks. (Arq Bras Endocrinol Metab 2007;51/6:938-942)

Keywords: Menopause; Hormone therapy; Climacterium 
$\mathrm{E}$ MBORA A REPOSIÇÃO ESTROGÊNICA esteja disponível há mais de 6 décadas, as mulheres e mesmo os profissionais da saúde estão confusos pelas opiniões divergentes em relação aos riscos e benefícios da terapia hormonal na menopausa (THM), estrogênica ou estro-progestagênica. A publicação de estudos randomizados e controlados, salientando-se o estudo HERS (Heart and Estrogen Replacement Study) (1) e o WHI (Women's Health Initiative) (2), intensificaram a controvérsia risco versus benefício. Milhões de mulheres são tratadas com THM para alívio da sintomatologia menopausal, incluindo sintomas vasomotores "fogachos" com sudorese intensa, nos quais o estrógeno é indiscutivelmente a terapêutica mais eficiente. Outras podem manter a terapia por longos períodos com o intuito de prevenir doenças crônicas. A preservação da massa óssea e a subseqüente redução do risco de fratura com a reposição estrogênica continuada isolada (3) ou associada a progestágeno (4) já está bem estabelecida.

Estudos observacionais sobre os efeitos vasculares e metabólicos dos estrógenos têm sugerido um benefício potente na redução de doenças cardiovasculares (5), mas nos resultados de estudos randomizados controlados publicados nos últimos anos, a reposição estrogênica não se mostrou benéfica em mulheres com doença vascular estabelecida nem em mulheres saudáveis. O aumento de risco de câncer de mama e doença tromboembólica também tem sido verificado nesses estudos. O encerramento prematuro de um dos braços do estudo WHI acarretou várias preocupações entre médicos, pacientes e profissionais da indústria farmacêutica. Existem, porém, dificuldades em extrapolar os resultados desses estudos, os quais usaram um produto especifico de reposição, numa população de mulheres também específicas, quando dispomos atualmente de várias formulações, doses e vias de administração diferentes. Entretanto, na ausência de evidências de que outros produtos são seguros, os dados sugerem que, para muitas mulheres, os riscos da utilização da $\mathrm{TH}$ prolongada superam os benefícios. A incidência absoluta dos eventos adversos é baixa, e o risco individual num ano é muito baixo, mas os riscos são cumulativos ao longo do tempo. $\mathrm{O}$ balanço risco-benefício em cada mulher sob uso continuado de TH exige avaliação permanente.

Nos três maiores estudos, que suscitaram todas as dúvidas, medos e controvérsias a respeito da terapia hormonal na menopausa, a média de idade das participantes no início da $\mathrm{TH}$ foi 60 anos, contribuindo para a idéia de que esses resultados não podem ser aplicados para aquelas que iniciaram a reposição no início da menopausa. A propósito, o braço do estudo WHI que utilizou reposição estrogênica isolada, sem adição de progestágeno, encontrou no subgrupo de mulheres mais jovens (50-59 anos) uma resposta mais favorável ao estrógeno quando comparada à resposta das mais idosas para vários endpoints (6). De uma maneira geral, o risco absoluto para várias doenças duplica em cada década da vida. Assim, mulheres com 50 anos de idade têm cerca de metade do risco daquelas com 60, e 1/4 do risco daquelas com 70 anos de idade. Isso significa que os riscos da terapia de reposição na menopausa podem ser bem menores na mulher jovem. Ou seja, há uma "janela de oportunidade" para a utilização da TH, situada principalmente no período de transição da menopausa.

Se o estudo WHI tivesse envolvido apenas mulheres de 50 a 55 anos, os resultados teriam sido os mesmos? Lobo (7) respondeu essa questão numa publicação envolvendo 4.000 pacientes, idade média de 53,6 anos, sendo 2.173 delas fazendo uso de estrógeno eqüino conjugado, $0,625 \mathrm{mg} / \mathrm{dia}$, associado ou não ao acetato de medroxiprogesterona. A amostra populacional de Lobo era 1,5 vezes maior do que a população similar do WHI. Lobo realizou a análise comparativa entre os grupos, guiando-se pelos mesmos parâmetros do WHI. As pacientes foram avaliadas por um ano. Observou que o número de eventos cardiovasculares/1000 pacientes/ano, incluindo acidente vascular cerebral, embolia pulmonar, trombose venosa profunda e infarto do miocárdio, no grupo submetido à terapêutica hormonal foi de 1,96 contra 3,01 do grupo placebo. Esses achados com certeza representam uma porta para outros estudos similares, abrangendo mulheres no período peri menopausa, incluídas na janela de oportunidade. As conclusões poderão ser muito diferentes das apresentadas nos grandes ensaios clínicos citados. Em estudo recente, visando análise da ocorrência de eventos cardiovasculares em uma população mais jovem do que estudo WHI, observou-se que entre as mulheres com idade entre 50-54 anos ocorreram 8 eventos cardiovasculares no grupo tratado contra 16 do placebo. Além disso, no grupo de mulheres com idade entre 55-59 anos, a ocorrência foi de 13 eventos contra 20 do grupo placebo (8), reforçando a idéia da idade correta para inicio da terapêutica hormonal de reposição.

No estado atual dos conhecimentos, não existem evidências que justifiquem o uso de TH para prevenção primária ou secundária de doenças cardiovasculares (DCV). Entretanto, também não existem estudos conclusivos a respeito de prevenção 
primária de DCV em mulheres na peri ou pós-menopausa recente. Os grandes estudos controlados, até então publicados, utilizaram um único regime terapêutico, caracterizado por doses convencionais, em pacientes assintomáticas e com faixa etária superior a 60 anos.

As sociedades médicas alteraram seus consensos e recomendam que a terapia de reposição na menopausa deva ser usada somente como tratamento da sintomatologia da menopausa (9-13).

\section{BENEFÍCIOS DEFINIDOS DA TH}

São considerados benefícios definidos da $\mathrm{TH}$ aqueles oriundos de estudos randomizados, com grupo controle e duplo cego, categoria I em evidência clinica.

\section{Sintomas vasomotores}

O tratamento dos fogachos e das alterações do sono acarretados pelos distúrbios vasomotores constitui a principal indicação de TH (14). Todos os tipos de TH (tipo de estrogênio, doses, via de administração com ou sem adição de progestágeno) são eficazes. Em uma revisão sistemática publicada pelo Instituto Cochrane, envolvendo 21 estudos randomizados e duplo-cegos sobre o efeito da $\mathrm{TH}$ comparada a placebo, totalizando 2.511 pacientes, verificou-se $77 \%$ de melhora dos fogachos nas usuárias de $\mathrm{TH}$ comparada a uma melhora de $30 \%$ no grupo placebo (15).

\section{Sintomas urogenitais}

O hipoestrogenismo é responsável por várias manifestações urogenitais na mulher menopausada: ressecamento vaginal, dispareunia, prurido vulvar, leucorréia, dificuldades de esvaziamento vesical, urgência e aumento da freqüência miccional, noctúria, disúria e incontinência urinária. Vários elementos são responsáveis pela continência urinária, incluindo aqueles estrogênio-dependentes, em particular o tônus e o trofismo do assoalho pélvico e da mucosa uretral. Também existem receptores de estrógeno e de progesterona no trato urinário inferior e assoalho pélvico nos ligamentos e nas fáscias musculares que compõem o diafragma pélvico e urogenital da mulher.

Todos os tipos de TH local ou sistêmica são eficazes no tratamento de sintomas de atrofia vulvar e vaginal. Em uma revisão sistemática avaliando o efeito preventivo da reposição estrogênica sobre infecções urinárias de reposição, observou-se que o estrogênio por via oral reduziu a freqüência de infecções urinárias $(\mathrm{OR}=2,51)$ comparado ao grupo placebo (16). Em outra metanálise, realizada pelo Grupo Cochrane, constituída de vários ensaios clínicos controlados empregando TH sistêmica ou local, foi demonstrado que a reposição pode melhorar a incontinência, particularmente a de urgência. Aproximadamente 50\% das pacientes em $\mathrm{TH}$ nos ensaios clínicos curaram ou melhoraram, comparadas a $25 \%$ de melhora entre os grupos placebo (16).

\section{Osteoporose}

Reposição estrogênica sistêmica isolada ou associada a progestágenos está aprovada nos Estados Unidos e Canadá para prevenção, mas não para tratamento da osteoporose da pós-menopausa. Porém, é consenso na literatura a eficácia da TH na manutenção da massa óssea e na prevenção de fraturas osteoporóticas (18). Mesmo o emprego de TH em baixas doses já se mostrou eficaz para o esqueleto $(19,20)$. Estudos têm mostrado a perda de 3 a $6 \%$ de massa óssea no primeiro ano de suspensão da TH sistêmica (18). Embora a indicação primária da $\mathrm{TH}$ seja o tratamento dos sintomas menopausais, quando na paciente que faz uso de TH melhoram ou terminam os sintomas devese considerar a manutenção da $\mathrm{TH}$ pelo seu efeito no osso, sempre reavaliando riscos e benefícios.

\section{Sintomas psíquicos}

Os efeitos protetores do estrógeno no cérebro incluem aumento da atividade colinérgica, redução da perda neuronal e estimulação axonal, proliferação de formações dendritícas, redução da isquemia cerebral por aumento da circulação e redução dos níveis de colesterol e modulação da expressão gênica da apolipoproteína E (21). As evidências que sustentam a hipótese de que a deficiência estrogênica seja uma das causas de demência senil é oriunda da associação positiva entre o estrógeno exógeno e a performance cognitiva em mulheres idosas sem demência. Estudos prospectivos têm demonstrado um risco de demência menor em mulheres usuárias de TH comparadas às não usuárias de reposição (22). Duas metanálises de estudos prospectivos observacionais concluíram redução de $29 \%$ (23) e 34\% (24) do risco de demência em mulheres com TH. Por outro lado, em estudos randomizados em mulheres portadoras de Doença de Alzheimer, o estrógeno não mostrou benefício na performance cognitiva, embora a maioria desses estudos tenha recebido várias críticas metodológicas (25).

Os estrogênios são encontrados em várias áreas do cérebro e estimulam o crescimento dos processos neuronais e conexões sinápticas com efeitos positivos sobre tamanho, número, conectividade, volume e plasticidade dos neurônios. Boccardi e cols. (26), em pu- 
blicação recente, avaliaram o efeito do estrógeno no volume de massa cinzenta em mulheres menopausadas sob reposição, através de ressonância magnética tridimensional de alta resolução. A estrogenoterapia pode diminuir a perda de massa cinzenta da pós-menopausa, as estruturas que exibiram maior volume em associação com a TH foram cerebelo, córtex cerebral, tipicamente envolvidas na Doença de Alzheimer, estruturas temporais mediais e parietais (26). O efeito neuroprotetor do estrógeno tem sido demonstrado in vivo e in vitro e pode ser atribuído à ativação dos receptores estrogênicos $\alpha$ e $\beta$, bem como sua capacidade de estimular a proliferação de células nervosas (26).

Harlow e cols. (27) acompanharam 996 mulheres na pré-menopausa por 3 anos consecutivos, com avaliações semestrais da sintomatologia psíquica, alterações hormonais e características dos ciclos menstruais. Neste estudo foi observado que mulheres com antecedentes depressivos desenvolveram mais alterações hormonais ao longo do seguimento, sendo a depressão um fator de risco associado ao desenvolvimento precoce de características perimenopausais, como os sintomas vasomotores e as irregularidades menstruais (27). O uso da estrogenoterapia tem sido associado à melhora dos sintomas depressivos em mulheres na perimenopausa. A progesterona ou progestogênios, usados isoladamente ou combinados com estrogênio, estão associados com menores reduções no humor deprimido (28).

\section{DOSE E VIAS DE ADMINISTRAÇÃO}

Sabe-se que muitos dos efeitos benéficos e adversos dos hormônios são dose-dependente. Portanto, há uma tendência na redução das doses hormonais nos atuais esquemas de $\mathrm{TH}$, o que poderá trazer novas perspectivas de redução dos riscos relacionados ao seu uso, de forma semelhante ao ocorrido com os contraceptivos orais.

São inúmeras as vias de administração da $\mathrm{TH}$, estando cada uma delas indicada caso a caso. A via oral difere da não oral quanto à passagem hepática, o que pode ser vantajoso ou contra-indicado em alguns casos. Existem evidências de que a via transdérmica não aumenta os níveis de proteína C-reativa e pode estar associada a um risco menor de trombose que a via oral.

\section{PROGESTÁGENOS}

Os progestágenos devem ser adicionados ao estrogênio em todas as mulheres menopausadas não histerectomizadas com o intuito de prevenir a hiperplasia e o adenocarcinoma endometrial estrógeno-induzidos. São vários tipos de progestágenos disponíveis com efeitos distintos; dá-se preferência à utilização da progesterona natural, porém cada caso deve sempre ser analisado individualmente.

\section{CONCLUSÃO}

A principal indicação para terapêutica hormonal na menopausa é o alívio dos sintomas menopausais, tais como sintomas vasomotores e alterações gênito-urinárias, e a prevenção de osteoporose nas pacientes de risco.

É fundamental a introdução da terapia no momento certo, segundo o conceito da "janela de oportunidade", período que se refere aos primeiros três anos pós-menopausa, onde os benefícios cardiovasculares e cognitivos podem ser observados. O tratamento por mais de 5 anos não adiciona risco significativo para câncer de mama, mas diminui significativamente o risco de fratura osteoporótica. Algumas mulheres podem ser mais susceptíveis ao risco de eventos tromboembólicos precoces, mas quando a $\mathrm{TH}$ for adequada após avaliação individualizada, os benefícios superam os riscos e o tratamento deve ser recomendado. Estudos futuros são necessários para identificar novas indicações para $\mathrm{TH}$ e diminuir ou abolir seus riscos. A pesquisa clínica continua na identificação de fatores genéticos que possam influenciar a resposta individual à $\mathrm{TH}$, diferentes formulações estrogênicas, diferentes vias de administração e liberação, além das opções de dose. Os médicos devem sempre fazer suas decisões terapêuticas com base nos riscos e benefícios individuais de cada paciente, tendo a responsabilidade e o dever de promover as condições para a mulher atravessar a transição menopáusica com qualidade de vida.

\section{REFERÊNCIAS}

1. Hulley S, Grady D, Bush T, Furberg C, Herrington D, Riggs B, et al. Randomized trial of estrogen plus progestin for secondary prevention of coronary heart disease in postmenopausal women. JAMA 1998;280:605-13.

2. Rossouw JE, Anderson GL, Prentice RL, LaCroix AZ, Kooperberg $C$, Stefanick ML, et al . Risks and benefits of estrogen plus progestin in healthy post-menopausal women: principal results from the Women's Health Initiative randomized controlled trial. JAMA 2002;288:321-33.

3. Lindsay R, Gallagher JC, Kleerekoper M, Pickar JH. Effect of lower doses of conjugated equine estrogens with and without medroxyprogesterone acetate on bone in early postmenopausal women. JAMA 2002;287:2668-76.

4. Cauley JA, Robbins J, Chen Z, Cummings SR, Jackson RD, LaCroix AZ, et al. Effects of estrogen plus progestin on risk of fracture and bone mineral density. The Women's Health Initiative Randomized Trial. JAMA 2003;290(3):1729-38. 
5. Grodstein F, Stampfer MJ, Manson JE, Colditz GA, Willett WC, Rosner B, et al. Postmenopausal estrogen and progestin use and the risk of cardiovascular disease. N Engl J Med 1996;335:453-61.

6. Anderson GL, Limacher M, Assaf AR, Bassford T, Beresford SA, Black H, et al.; Women's Health Initiative Steering Committee. Effects of conjugated equine estrogen in postmenopausal women with hysterectomy. The Women's Health Initiative Randomized Controlled Trial. JAMA 2004;291(14):1701-12.

7. Lobo RA. Evaluation of cardiovascular event rates with hormone therapy in healthy, early postmenopausal women. Arch Intern med 2004;164:482-4.

8. Hsia J, Langer R, Manson JE, Kuller L, Johnson KC, Hendrix $\mathrm{SL}$, et al. Conjugated equine estrogens and coronary heart disease: The Women's Health Initiative. Arch Int Med 2006;166(3):357-65.

9. Utian WH, Collins P, Ettinger B, Gallagher JC, Gass MLS, Gelfand MM, et al. Estrogen and progestogen use in peri and postmenopausal women: September 2003 position statement of The North American Menopause Society. Menopause 2003;10(6):497-504.

10. Skouby SO, Azzawi FA, Barlow D, Ertungealp JCAE, Gompel A, Graziottin A, et al. Climacteric medicine: European Menopause and Andropause Society (EMAS) 2004/2005 position statements on peri and postmenopausal hormone replacement therapy. Maturitas 2005;51:8-14.

11. Naftolin F, Schneider HPG, Sturdee DW. Guidelines for hormone treatment of women in the menopausal transition and beyond. Position Statement by the executive Committee of the International Menopause Society. Maturitas 2005;51:15-20.

12. Fernandes $C E$, Soares Pereira $A$, Machado $R B$, et al. Consenso da SOBRAC - Associação Brasileira de Climatério. Terapêutica hormonal na peri e na pós-menopausa. São Paulo 2004: Medcomm.

13. Clapauch R, Athayde A, Meirelles R, Weiss RV, Pardini DP, Leão LMC, et al.Terapia de reposição hormonal na menopausa .Posicionamento do Departamento de Endocrinologia Feminina e Andrologia da SBEM. Arq Bras Endocrinol Metab 2005;49(3):449-54.

14. Santoro NF, Clarkson TB, Freedman RR. Treatment of menopause-associated vasomotor symptoms: position statement of The North American Menopause Society. Menopause 2004;11:11-33.

15. MacLennan A, Lester S, Moore V. Oral oestrogen replacement therapy versus placebo for hot flushes (Cochrane Review). The Cochrane Library, Issue 1. Chichester: John Wiley \& Sons, Ltd., 2004.

16. Cardozo L, Lose G, McClish D, Versi E, Koning GH. A systematic review of estrogens for recurrent urinary tract infections: third report of the hormones and urogenital therapy committee. Int Urogynecol J Pelvic Floor Dysfunct 2001;12:15-20.
17. Moehrer B, Hextall A, Jackson S. Oestrogens for urinary incontinence in women (Cochrane Review). The Cochrane Library, Issue 1. Chichester: John Wiley \& Sons, Ltd., 2004.

18. Management of osteoporosis in postmenopausal women: 2006 position statement of The North American Menopause Society. [Position Statement] Menopause 2006;13(3):34067.

19. Lindsay R, Gallagher JC, Kleerekoper M, Pickar JH. Effect of lower doses of conjugated equine estrogens with and without medroxyprogesterone acetate on bone in early postmenopausal women. JAMA 2002;287:2668-76.

20. Prestwood KM, Kenny AM, Kleppinger A, Kulldorff $M$. Ultralow-dose micronized $17 \alpha$-estradiol and bone density and bone metabolism in older women: a randomized controlled trial. JAMA 2003;290:1042-8.

21. Shumaker $S$, Legault $C$, Rapp $S$, Thal $L$, Wallace $R$, Robert $B$ et al. Estrogen plus progestin and the incidence of dementia and mild cognitive impairment in postmenopausal women: The Women's Health Initiative memory study: A randomized controlled trial. JAMA 2003;289(20):2651-62.

22. Tang MX, Jacobs D, Stern Y, Marder K, Schofield P, Gurland $B$, et al. Effect of estrogen during menopause on risk and age at onset of Alzheimer disease. Lancet 1996;348:429-32.

23. Yaffe K, Sawaya G, Lieberburg I, Grady D. Estrogen Therapy in postmenopausal women: effects on cognitive function and dementia. JAMA 1998;279:688-95.

24. Nelson HD, Humphrey LL, Nygren P, Teutsch SM, Allan JD. Postmenopausal hormone replacement: scientific review. JAMA 2002;288:872-81.

25. Shumaker SA, Reboussin BA, Espeland MA, Rapp SR, McBee WL, Dailey M, et al. The Women's Health Initiative Memory Study (WHIMS): a trial of the effect of estrogen therapy in preventing and slowing the progression of dementia. Control Clin Trials 1998; 19:604-21.

26. Boccardi M, Ghidoni R, Govoni S, Testa C, Benussi L, Bonetti $M$, et al. Effects of hormone therapy on brain morphology of healthy postmenopausal women: a Voxel based morphometry study. Menopause 2006;13(4):584-91.

27. Harlow BL, Wise LA, Otto MW, Soares CN, Cohen LS. Harvard study of moods and cycles. Arch Gen Psychatry 2003;60:29-36.

28. Zweifel JE, O'Brien WH. A meta-analysis of the effect of hormone replacement therapy upon depressed mood. Psychoneuroendocrinology 1997;22:189-212.

\section{Endereço para correspondência:}

Dolores Pardini

Rua Júpiter 160, apto. 111

01532-030 São Paulo, SP 